J. Phys.: Condens. Matter 24 (2012) 052203.

http://dx.doi.org/10.1088/0953-8984/24/5/052203

\title{
Hydrogen-induced blistering mechanisms in thin film coatings
}

\author{
A S Kuznetsov ${ }^{1}$, M A Gleeson ${ }^{1}$, and F Bijkerk ${ }^{1,2}$. \\ ${ }^{1}$ FOM Institute for Plasma Physics Rijnhuizen, Postbus 1207, 3430 BE Nieuwegein, The \\ Netherlands. (www.rijnhuizen.nl) \\ ${ }^{2}$ MESA+ Institute for Nanotechnology, University of Twente, Postbus 217, 7500 AE Enschede, \\ The Netherlands. \\ E-mail: F.Bijkerk@rijnhuizen.nl
}

\begin{abstract}
We report on the mechanisms of hydrogen-induced blistering of multilayer coating. The blister formation is a result of highly-localized delamination occurring at the two outermost metalon-silicon interfaces. The number, size, and type of blisters formed varied depending on the composition and ion energy of the incident flux. The results are explained in terms of the multilayer structure being simultaneously susceptible to blistering via two independent mechanisms. A high density of small blisters developed when relatively energetic (several 100’s $\mathrm{eV}$ ) ions were present. Independently, a hydrogenation process that was facilitated by the presence of a small flux of low energy ions $(\leq 50 \mathrm{eV})$ induced a low density of large blisters.
\end{abstract}

PACS: 34.35.+a; 61.80.Jh; 68.35.Gy; 68.65.Ac; 81.40.Np; 81.40.Wx.

\section{Introduction}

Blistering and delamination of materials as a result of direct exposure to energetic (keV) ions is a wellknown phenomenon [1-3]. The effect is typically associated with stress relief, with the blister formation being localized in a region determined by the mean ion penetration depth. Comparable effects can also be induced with the aid of suitable defect/trap layers. For instance, high energy ion irradiation and implantation followed by low energy plasma-driven hydrogenation has been used to induce delamination of silicon [4-6]. Blistering and the associated buckling and delamination of deposited thin films [7-9] is also associated with the relaxation of stress [10-12]. Hence, a buried compressively strained layer has been used to induce controlled delamination of thin Si layers [13, 14], with the strained layer acting initially as a preferential trap for vacancies leading ultimately to highly-localized crack formation. 


\section{J. Phys.: Condens. Matter 24 (2012) 052203. \\ http://dx.doi.org/10.1088/0953-8984/24/5/052203}

There have been some reports on blistering related specifically to multilayer (ML) systems [15-17]. These studies have focused on the formation of blisters under high-energy (33-100 keV) He ion irradiation and are aimed at the development of damage resistant materials for use in harsh environments such as fusion reactors. More recently, damage to ML coatings of a type intended for solar mission instrumentation has been reported as a result of $1 \mathrm{keV}$ hydrogen ion bombardment [18]. Layer detachment resulting in a degradation of the optical performance was observed. Similar damage may be induced as a result of bombardment by solar wind particles (see [18] and references therein). It is necessary to fully understand the processes that underpin such effects in order to facilitate the development of MLs intended for use in such potentially hazardous conditions.

We have investigated the susceptibility of the latter type of ML structure to blistering by comparing the effects of a variety of hydrogen exposure conditions on uncapped Mo/Si ML samples. Various exposures resulted in the appearance of symmetric blisters on the sample surface. These were the result of highlylocalized bilayer delaminations occurring exclusively at the two outmost Mo-on-Si interfaces. The extent and type of blistering observed was strongly dependent on the composition of the incident hydrogen flux. The samples were unaffected by exposure to neutral hydrogen but exhibited blistering once ions were present. Two distinct blister size distributions are observed. On the basis of correlating the characteristics of the blisters formed with the composition of the incident flux, the ML structure is identified as being simultaneously susceptible to blistering via two separate mechanisms. One is induced by relatively energetic (e.g. $650 \mathrm{eV}$ ) ions, while the other is a hydrogenation process that is facilitated by the presence of low energy $(\leq 50 \mathrm{eV})$ ions generating a sub-nA current on the sample.

\section{Experimental}

The samples investigated were Si-terminated ML stacks consisting of $50 \mathrm{Mo} / \mathrm{Si}$ bilayers deposited on an ultra-smooth Si wafer. They were produced by magnetron sputtering, with post-deposition polishing of the individual Si layers by low energy $\mathrm{Kr}$ ions $[19,20]$. The layer thicknesses were $\sim 3 \mathrm{~nm}(\mathrm{Mo})$ and $\sim 4$ nm (Si). All samples were exposed to atmospheric conditions after deposition and again after hydrogen 


\section{J. Phys.: Condens. Matter 24 (2012) 052203. \\ http://dx.doi.org/10.1088/0953-8984/24/5/052203}

irradiation. All irradiations were performed in a single vacuum vessel, which had a typical base pressure of $\sim 1 \times 10^{-8}$ mbar. Samples were mounted on a manipulator that allowed radiative heated from the back side. No pre-treatment was applied to the samples before the hydrogen exposures.

Blistering has been observed after exposures using a variety of hydrogen sources. The results shown in this report are for a capillary-type thermal cracker (Oxford Applied Research TC 50); an ion sputter gun (Riber CI-10) and a hot W filament used in conjunction with an independently biased (+50 V) plate (henceforth denoted "W-fil/Plate"). The filament, sample, and plate formed a roughly triangular configuration. When biased, the plate attracted and accelerated electrons emitted from the filament. The resultant electron ionization produced a net positive current on the sample of $\sim 0.1 \mathrm{nA}$. Thus, the maximum energy that could be attained by ions reaching the grounded sample surface was $50 \mathrm{eV}$. The capillary cracker is nominally a neutral source. However, it utilizes electron emission heating of a $+1 \mathrm{keV}$ biased capillary resulting in the production of ions that generated a net positive current on the sample. For the current exposures the capillary cracker was operated at a power of $55 \mathrm{~W}$ with a $1 \mathrm{sccm}$ flow of $\mathrm{H}_{2}$. These conditions produced a current on the sample of $\sim 75 \mathrm{nA}$. Although the ions in the centre of the beam had a broad energy range $(0-1000 \mathrm{eV}$; measured with a retarding field analyzer), the majority of these ions had a relatively well-defined energy of $\sim 860 \mathrm{eV}$. For the ion gun exposures the ion energy was $\sim 650$ $\mathrm{eV}$ and the current generated was $\sim 3.9 \mu \mathrm{A}$. The experimental conditions specific to the individual exposures are tabulated later in the text when the effects observed for different sources are compared.

\section{Results}

Damage to the samples as a result of hydrogen exposure was first evident as a dulling of the original highly-reflective surface that was visible to the naked eye before breaking vacuum (sometimes requiring the aid of a strong light). Atomic force microscopy (AFM) revealed the damage to be due to blister-like structures on the surface. Figure 1(a) shows a 3-D representation of a $25 \times 25 \mu \mathrm{m}^{2}$ AFM scan obtained from a sample after a 5 hour exposure using the capillary cracker. It contains $\sim 450$ distinct features. Figure 1(b) shows a 2-D representation of a $5 \times 5 \mu \mathrm{m}^{2}$ scan from the same sample illustrating the highly 


\section{J. Phys.: Condens. Matter 24 (2012) 052203. \\ http://dx.doi.org/10.1088/0953-8984/24/5/052203}

symmetric nature of the structures formed. In addition to single blisters, several instances of overlapping double-blister structures are visible. Two distinct blister sizes are evident in both images. The majority of blisters have a height of 35-65 $\mathrm{nm}$ and a diameter of 300-500 $\mathrm{nm}$. These are interspersed with a lower number of large blisters that have heights up to $\sim 180 \mathrm{~nm}$ and diameters up to $\sim 1.7 \mu \mathrm{m}$.
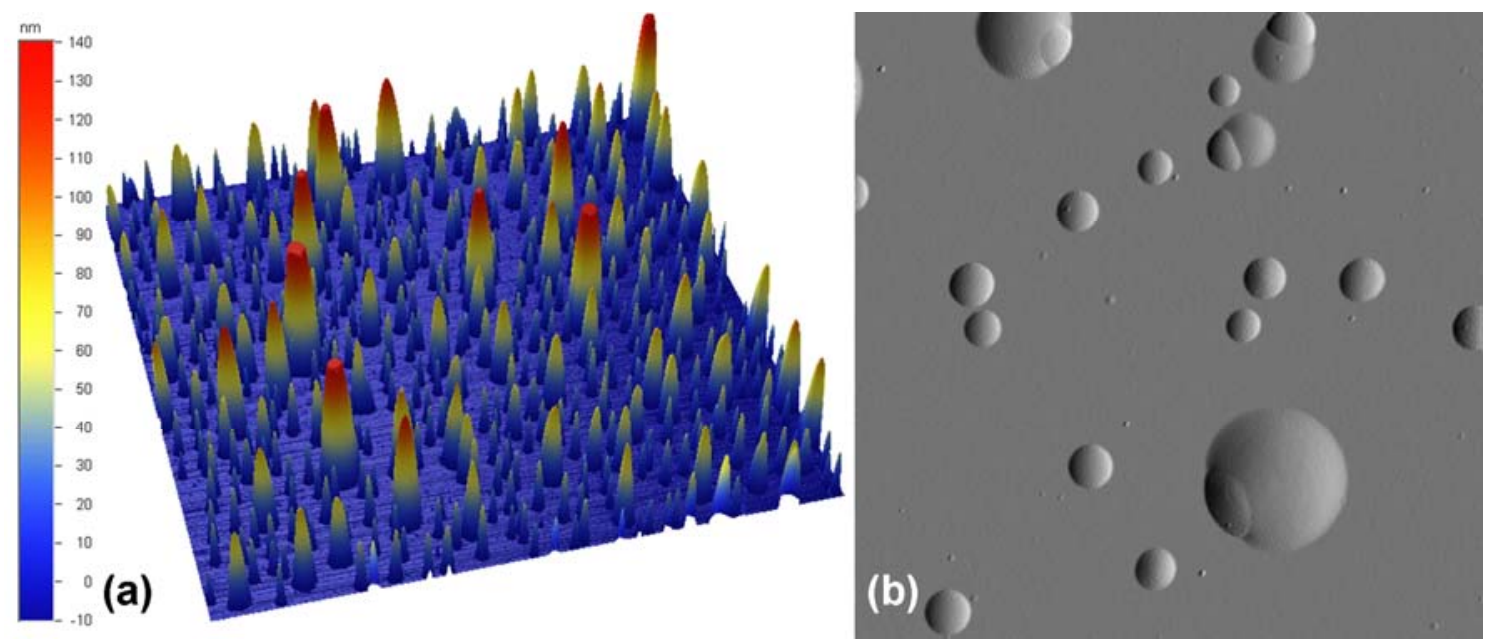

Figure 1. AFM images collected from a sample after 5 hours exposure via the capillary cracker. (a) 3-D representation of a $25 \times 25 \mu \mathrm{m}^{2}$ region. (b) $2-\mathrm{D}$ representation of a $5 \times 5$ $\mu \mathrm{m}^{2}$ region.

Cross-sectional transmission electron microscopy (TEM) revealed the blisters to be the result of localized detachments occurring exclusively near the two outermost Mo-on-Si interfaces of the ML stack. Representative TEM images from the sample after 5 hours exposure using the capillary cracker are shown in figure 2. The dark (light) layers are Mo (Si). Figure 2(a) illustrates the origin of the double-blister structures seen in figure 1(b). They arise from overlapping delaminations occurring at different interfaces. The larger blister is due to single-bilayer detachment while the smaller blister is due to double-bilayer detachment. Figure 2(b) shows a cross-section of a complete blister due to single-bilayer detachment. Figure 2(c) shows a close-up of the detachment point of a double-bilayer blister. Detachment at deeper interfaces and detachment at Si-on-Mo interfaces have not been observed. 
J. Phys.: Condens. Matter 24 (2012) 052203.

http://dx.doi.org/10.1088/0953-8984/24/5/052203
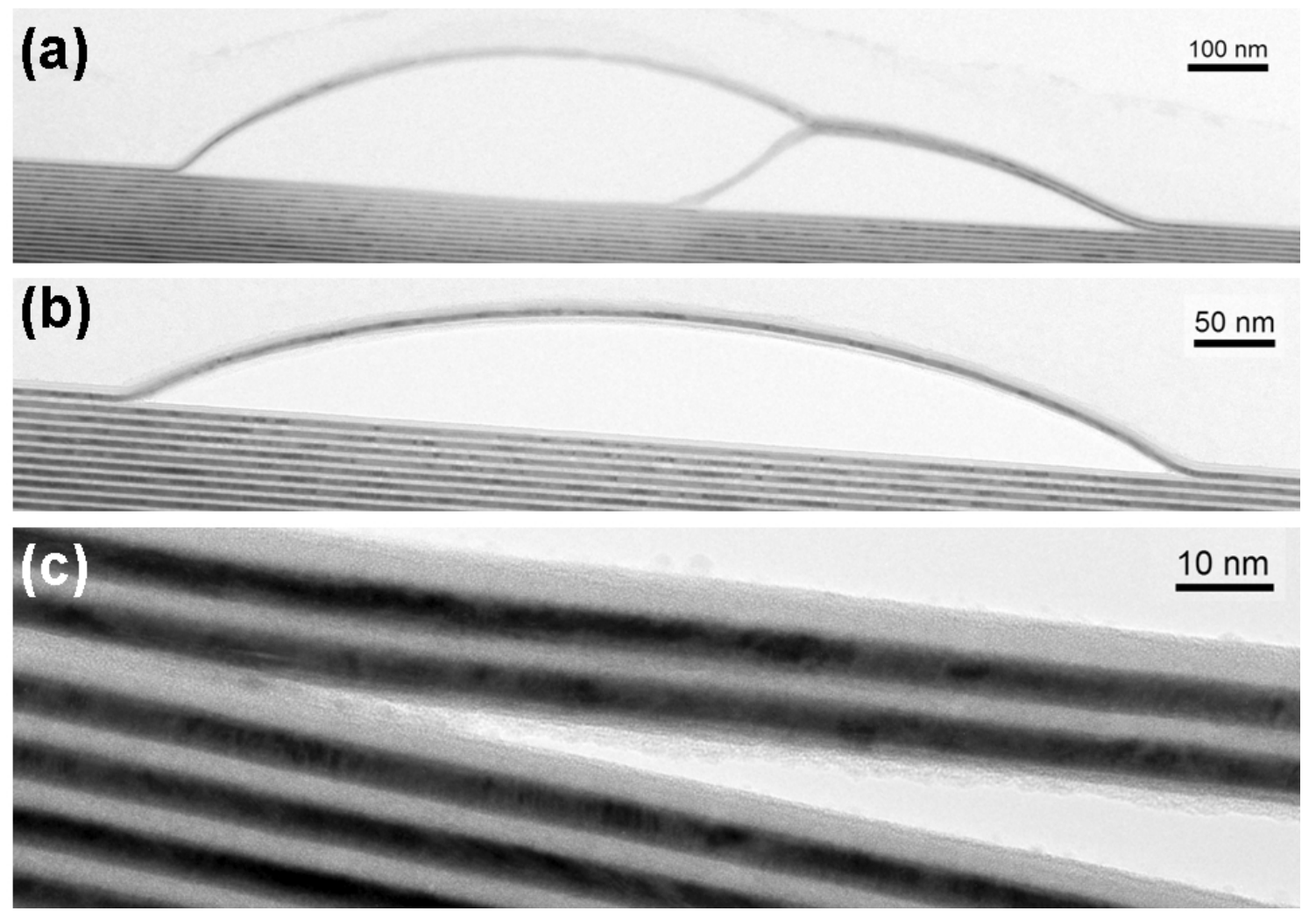

Figure 2. TEM cross-sections collected from a sample after 5 hours exposure via the capillary cracker. (a) Double-blister; detachments at $1^{\text {st }}$ and $2^{\text {nd }}$ Mo-on-Si interface. (b) Single-blister; detachment at $1^{\text {st }}$ Mo-on-Si interface. (c) Close-up of a double-bilayer detachment point.

Blisters developed on the current samples after hydrogen exposure using the three sources listed in the experimental section. A common feature of all exposures that resulted in blister formation was the presence of ions in the irradiating flux. In this report we illustrate the results of various exposure conditions and attempt to correlate them with the likely mechanisms involved. The conditions applicable to the individual exposures are listed in table 1.

Table 1. Exposure conditions utilized in the current work.

\begin{tabular}{ccccc}
\hline Source & $\begin{array}{c}\text { Exposure } \\
\text { time (s) }\end{array}$ & $\begin{array}{c}\text { Pressure }\left[\mathrm{H}_{2}\right] \\
(\mathrm{mbar})\end{array}$ & $\begin{array}{c}\text { Sample } \\
\text { temperature }(\mathrm{K})\end{array}$ & $\begin{array}{c}\text { Sample } \\
\text { current (nA) }\end{array}$ \\
\hline Capillary cracker & 10800 & $5 \times 10^{-5}$ & $300-360$ & 75 \\
Capillary cracker & 18000 & $5 \times 10^{-5}$ & $300-360$ & 75 \\
W-fil/Plate & 3600 & $2 \times 10^{-2}$ & $300-390$ & 0.1 \\
Ion Gun & 3600 & $5 \times 10^{-5}$ & 373 & 3900 \\
\hline
\end{tabular}




\section{J. Phys.: Condens. Matter 24 (2012) 052203. \\ http://dx.doi.org/10.1088/0953-8984/24/5/052203}

The elevated temperatures indicated in table 1 for the capillary cracker and W-fil/Plate exposures are the result of direct heating by the respective sources and the absence of active sample cooling. In the specific case of the $\mathrm{W}$-fil/Plate, once the filament was switched on the sample temperature quickly exceeded 350 $\mathrm{K}$ and continued to increase at a more gradual rate over the course of the exposure. Due to the extended time used for the capillary cracker exposures, the temperature was stable at the maximum indicated for most of the exposure. There was no significant direct heating of the sample by the ion gun. Deliberately heating was applied during this exposure in order to maintain the sample temperature in a range comparable to that produced by the other sources. Blistering at room temperatures has been observed for exposures using the (retracted) capillary cracker and the ion gun. The details of the temperature dependence of the blister formation are currently under investigation. In general, visible damage to the sample surface was observed to develop faster at higher temperatures. In addition, with increasing temperature the number of blisters formed decreases, while the size of individual blisters increases. The exposures performed at room temperature with the ion gun and the capillary cracker both resulted in high densities of very small blisters. Tentatively, the development of the larger blister distribution evident in figure 1 appears to be suppressed as the temperature is reduced.

Figure 3 shows a comparison of $25 \times 25 \mu \mathrm{m}^{2}$ AFM images obtained after the various exposures indicated in table 1. All images are represented on a common Z-scale. Figure 3(a)\&(b) were obtained after exposure via the capillary cracker for 3 and 5 hours respectively. Figure 3(c) is the result of the 1 hour exposure to W-fil/Plate (50 V bias) in a hydrogen atmosphere. No damage was evident when a similar exposure was performed without the biased plate. Finally, figure 3(d) is the result of the 1 hour hydrogen exposure via the ion gun. 


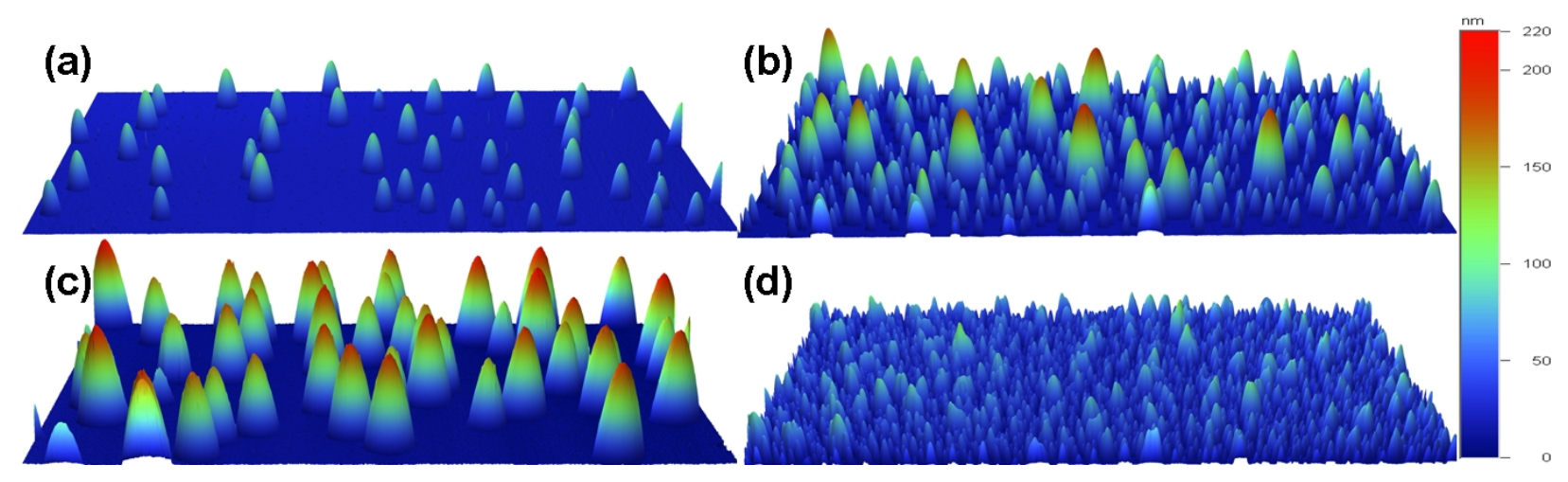

Figure 3. 3-D representations of $25 \times 25 \mu \mathrm{m}^{2}$ AFM scans from samples exposed for: (a) 3 hours and (b) 5 hours via the capillary cracker; (c) 1 hour to the W filament with $+50 \mathrm{~V}$ biased plate; (d) 1 hour via the ion gun.

The 3 hour exposure to the capillary cracker resulted in a low density of moderately sized blisters (as compared with the 5 hour exposure). As already shown in figure 1, a high density of small blisters interspersed with larger blisters is present after 5 hours. The W-fil/Plate exposure produced a low density of large blisters after 1 hour. This source produced the largest blisters of the exposures shown. The ion gun produced a very high density of predominately small blisters after 1 hour. Table 2 gives an estimate of the composition and total fluence of particles incident on the sample surface for these exposures.

Table 2. Exposure composition estimates associated with figure 3.

\begin{tabular}{ccccccc}
\hline \multirow{2}{*}{ Source } & \multirow{2}{*}{$\begin{array}{c}\text { Image } \\
\text { [figure 3] }\end{array}$} & \multicolumn{3}{c}{ Fluence estimate $\left(\mathrm{cm}^{-2}\right)$} & \multirow{2}{*}{$\begin{array}{c}\text { Ion } \\
\text { fraction }\end{array}$} & $\begin{array}{c}\text { Ion } \\
\text { energy } \\
\text { (eV) }\end{array}$ \\
Capillary cracker & (a) & $4.8 \times 10^{21}$ & $2.4 \times 10^{21}$ & $5.1 \times 10^{15}$ & $7.1 \times 10^{-7}$ & $0-1000$ \\
Capillary cracker & (b) & $7.9 \times 10^{21}$ & $4.0 \times 10^{21}$ & $8.4 \times 10^{15}$ & $7.1 \times 10^{-7}$ & $0-1000$ \\
W-fil/Plate & (c) & $3.6 \times 10^{20}$ & $7.7 \times 10^{22}$ & $2.2 \times 10^{12}$ & $2.9 \times 10^{-11}$ & $\leq 50$ \\
Ion Gun & (d) & - & $1.9 \times 10^{20}$ & $8.6 \times 10^{16}$ & $4.5 \times 10^{-4}$ & 650 \\
\hline
\end{tabular}

The ion fluence estimates are based on the drain current measured on the sample plate. For the W-fil/plate and ion gun sources, the $\mathrm{H}_{2}$ fluences have been derived from the operating pressure in the chamber during the exposures. In the case of the capillary cracker, the $\mathrm{H}_{2}$ fluence is based on the flow value used and the quoted cracking efficiency of the capillary $\left(50 \%\right.$ for $\left.\mathrm{H}_{2}\right)$. In this case, the contribution from the background gas has been neglected. The $\mathrm{H}$ fluence for the capillary cracker has also been derived from 


\section{J. Phys.: Condens. Matter 24 (2012) 052203. \\ http://dx.doi.org/10.1088/0953-8984/24/5/052203}

the flow value and quoted cracking efficiency. The $\mathrm{H}$ fluence estimate for the $\mathrm{W}$-fil/plate source is based on etch rate measurements using deposited carbon films. Note that all of these estimates represent an average over the total sample surface area. However, only the W-fil/Plate source produced damage that appeared uniform. Both the capillary cracker (collimated beam) and the ion gun (ion focusing) have nonuniform flux profiles that resulted in a visible variation in the damage pattern across the surface. For these sources the AFM scans shown in this work were collected in the region of maximum damage (beam centre).

Table 3 gives a blister-count and the fraction of the surface covered by blisters for the various exposures. Blisters are designated as "small" and "large" on the basis of their absolute size and aspect ratio (lateral radius:Z-height). Note that this designation is not specifically based on the absolute dimensions of the blisters but on the relative sizes of the features produced by a given exposure. "Large" blisters are present in all cases, whereas "small” blisters are primarily evident in figure 3(b)\&(d).

Table 3. Blister count and percentage coverages associated with figure 3.

\begin{tabular}{|c|c|c|c|c|}
\hline \multirow{2}{*}{ Source } & \multirow{2}{*}{$\begin{array}{c}\text { Image } \\
\text { [figure 3] }\end{array}$} & \multicolumn{2}{|c|}{ Blister count ${ }^{\mathrm{a}}$} & \multirow{2}{*}{$\begin{array}{c}\text { Blister } \\
\text { coverage (\%) }\end{array}$} \\
\hline & & Small & Large & \\
\hline Capillary cracker & (a) & 7 & 43 & 3.7 \\
\hline Capillary cracker & (b) & 378 & 72 & 17.2 \\
\hline W-fil/Plate & (c) & 0 & 38 & 13.8 \\
\hline Ion Gun & (d) & 3550 & 177 & 37.9 \\
\hline
\end{tabular}

a Blister counts are based on a $25 \times 25 \mu \mathrm{m}^{2}$ AFM scan area with the exception of the "small" blister count for the ion gun. Due to the high feature density, this count is extrapolated from a higher resolution $5 \times 5 \mu \mathrm{m}^{2}$ AFM image (142 small and 5 large blisters). In all cases, partial blisters appearing on image borders were not counted.

Figure 4 shows plots of the individual blister radius versus blister height derived from AFM images. The blisters that are designated in table 3 as "small” and "large" are indicated on this figure by filled and open circles, respectively. This illustrates the difference in blister aspect ratio that was used in distinguishing and quantifying the two distributions. "Large” blisters are somewhat flatter (larger radius for a given Z height) than "small” blisters. The "small” features indicated for the 3 hour capillary cracker exposure are at the limit of the lateral resolution of the AFM image. Hence the dimensions indicated in this case should 


\section{J. Phys.: Condens. Matter 24 (2012) 052203. \\ http://dx.doi.org/10.1088/0953-8984/24/5/052203}

be treated with caution. These features are currently attributed to the onset of "small” blisters, but they might also represent an early stage of "large” blisters development.
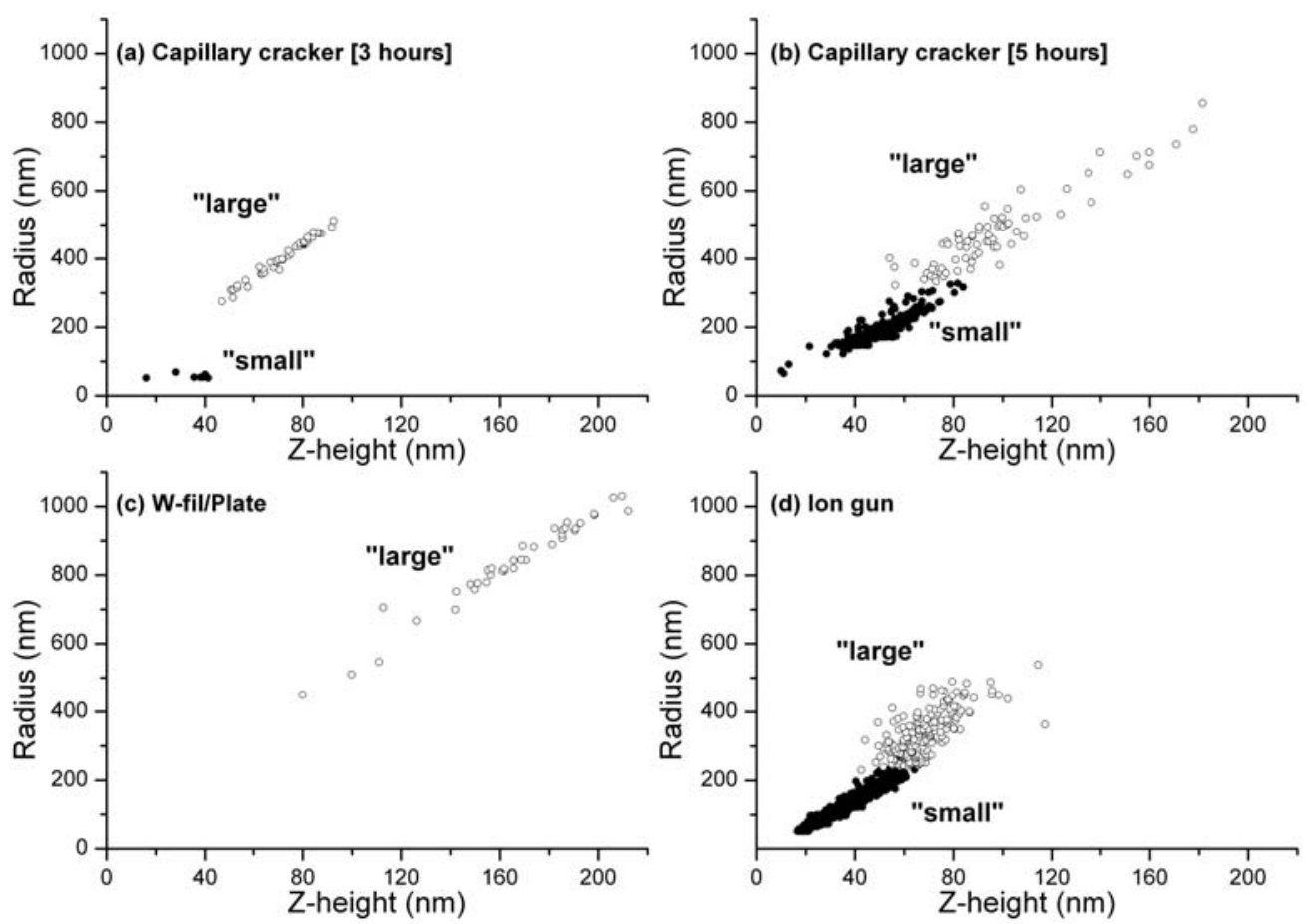

Figure 4. Plots of radius versus the corresponding Z-height of the individual blisters derived from the AFM scans shown in figure 3. Filled circles indicate blisters that were designated as "small” and open circles indicate blisters designated as "large".

\section{Discussion}

In the first instance, the hydrogen-induced blistering of MLs can be attributed to stress-induced delamination resulting from hydrogen incorporation into the stack (see literature cited in the introduction). The as-deposited Si and Mo layers in the current samples typically have compressive and tensile stress, respectively [21, 22]. Hence, layer stresses and defects that allow blistering to occur are introduced by the deposition processes rather than by specific post-deposition treatment of the sample. The localization of the delamination point can be understood on the basis of vacancy accumulation and subsequent hydrogen trapping effects introduced by compressively strained buried layers $[13,14]$. The Si layers in the current 


\section{J. Phys.: Condens. Matter 24 (2012) 052203. \\ http://dx.doi.org/10.1088/0953-8984/24/5/052203}

samples play the role of the buried SiGe layers in the previous works. They provide nucleation points for vacancies and incorporated hydrogen, leading ultimately to blister formation.

The unique aspect of the current results is that comparison of the images and correlation of the number and type of blisters formed with the exposure conditions clearly suggests the presence of two independent paths to blistering. One is induced by the presence of "energetic" ions and produced the high density of "small” blisters seen in figure 3(b)\&(d). The number of "small” blisters produced by these two exposures correlates with the (energetic) ion fluence (see tables 2 and 3), indicating that such ions have the potential to induce new preferential blister initiation sites. The need to create new initiation sites can be expected to introduce a fluence threshold to the appearance of the associated blistering. Such a threshold for the appearance of the "small” blisters is indeed suggested by the comparison of the two capillary cracker exposures (figure 3(a)\&(b)).

The other pathway to blister formation is a hydrogenation process that does not require energetic ions, Blistering induced by hydrogenation is typically dominated by the availability of pre-existing initiation sites since incident particles do not have sufficient energy to generate new initiation sites. In such cases, the number of blisters that can develop should be primarily related to the sample preparation methods and be relatively independent of the total fluence. The "large" blisters seen in figure 3(a)-(c) are attributed to this process. It is noteworthy that the 3 hour capillary cracker and W-fil/Plate exposures produced a roughly equivalent numbers of large blisters. The 5 hour capillary cracker and ion gun exposures produced noticeably more "large" blisters. However, in both of these cases the blister size analysis is complicated by the presence of double and complex multi-blister structures. This is reflected in the increased scatter in the aspect ratio of the "large" blisters produced by these exposures (see figure 4(b)\&(d)). It is noteworthy these double-/complex-blister structures are only evident on the samples that also exhibit the "small” blisters (i.e. once the influence of energetic ions has manifested). This suggests that there may be an inter-play between the ion-induced and hydrogenation processes. Such an inter-play would also explain the increased number of "large” blisters on these samples. 


\section{J. Phys.: Condens. Matter 24 (2012) 052203. \\ http://dx.doi.org/10.1088/0953-8984/24/5/052203}

The size of the individual large blisters produced by the various exposures is most directly correlated with the estimated $\mathrm{H}_{2}$ fluence. Exposures to date indicate that the main influence of the operating $\mathrm{H}_{2}$ pressure is on the growth rate of large blisters. Hence, the rapid development of very large blisters by the Wfil/plate exposure is a consequence of the high operating pressure. When this source is operated at significantly lower pressures ( $10^{-4}$ mbar range) it also produces a single distribution of "large” blisters, but these develop at a slower rate.

In the scheme outlined above, the ion gun induces blistering primarily via the ion-induced mechanism and the large ion flux quickly produces a high density of small blisters. For the W-fil/Plate only the hydrogenation pathway is open, limiting the number of blisters that form. The ionization pathway provided by the biased plate facilitates the hydrogenation process, but the ions generated do not lead to the creation of new preferential initiation sites. The presence of a high $\mathrm{H}_{2}$ background induces rapid blister growth as compared with the "large” blisters formed by the capillary cracker. Both mechanisms appear to be operative in the case of the capillary cracker. The features observed after 3 hours of exposure are primarily consistent with hydrogenation. After 5 hours the large blisters have increased in size, and somewhat in number. However, they are vastly outnumbered by the small blisters. Comparing individual blister shapes resulting from the different exposures indicates that when blistering via hydrogenation is dominant (W-fil/Plate; 3 hour capillary cracker) exclusively single blisters are formed. In contrast double/multiple-blister structures are common when the ion-induced process emerges (5 hour capillary cracker; ion gun). This difference is consistent with the proposed dual paths. The energetic ions present in the current exposures are capable of penetrating several bilayers, whereas the first available nucleation interface can act as a barrier to the hydrogenation process.

There are several noteworthy aspects of the current samples when compared with previous reports on the blistering of materials. One is the ability to efficiently development of blisters using basic laboratory vacuum equipment and relatively benign exposure conditions. This is in contrast to materials that may require high (keV) energies and/or plasma processing in order to induce blisters. Another is the discrete nature and highly self-consistent dimensions of the blisters formed. Equivalent blisters could be induced 


\section{J. Phys.: Condens. Matter 24 (2012) 052203. \\ http://dx.doi.org/10.1088/0953-8984/24/5/052203}

on different samples in a very reproducible fashion. These characteristics make such samples appealing as a model system for detailed investigation of blistering processes. Their potential in this regard is illustrated by the ability, as demonstrated in this manuscript, to clearly distinguish parallel (and potentially competing) processes.

\section{Conclusion}

We have demonstrated that hydrogen-induced blistering of ML samples can be strongly dependent on the exposure conditions. Blister formation, which is the result of delamination occurring at the Mo-on-Si interfaces of the two outermost bilayers in the stack, is attributed to a stress-driven delamination of hydrogen-implanted layers. Two distinct pathways to blistering are identified: “ion-induced” and "hydrogenation". The former is the direct result of the presence of relatively energetic (100’s eV) ions in the incident flux whereas the latter is facilitated by presence of low-energy $(\leq 50 \mathrm{eV})$ ions. The energetic ions can induce new blister initiation sites within the sample. In this case, the number of blisters formed is correlated with the ion fluence received by the sample and there is a threshold to the appearance of blisters. In contrast, the number of blisters formed via "hydrogenation" is dominated by pre-existing initiation sites that are introduced during the deposition process. The individual blister size in this case is correlated with the neutral hydrogen fluence. The results indicate that careful consideration must be given to the composition of the irradiating flux when analyzing the formation of blisters on such structures.

\section{Acknowledgements}

TEM sample preparation and imaging was done by Dr. Enrico G. Keim of the MESA ${ }^{+}$Institute, University of Twente. The 'Stichting voor Fundamenteel Onderzoek der Materie (FOM)', Utrecht is financially supported by the 'Nederlandse Organisatie voor Wetenschappelijk Onderzoek (NWO)'. The authors also acknowledge funding by AgentschapNL. 


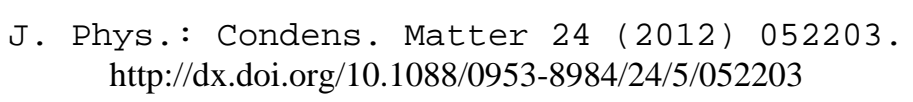

\section{References}

[1] Giguere A, Desrosiers N and Terreault B 2005 Appl. Phys. Lett. 87211911

[2] Goudeau P, George M and Coupeau C 2004 Appl. Phys. Lett. 84 894-6

[3] Lee J K, Lin Y, Jia Q X, Hochbauer T, Jung H S, Shao L, Misra A and Nastasi M 2006 Appl. Phys. Lett. 89101901

[4] Usenko A Y and Ulyashin A G 2002 Jpn. J. Appl. Phys. 41 5021-3

[5] Chen P, Chu P K, Hochbauer T, Lee J K, Nastasi M, Buca D, Mantl S, Loo R, Caymax M, Alford T, Mayer J W, Theodore N D, Cai M, Schmidt B and Lau S S 2005 Appl. Phys. Lett. 86031904

[6] Ma Y, Job R, Dungen W, Huang Y L, Fahrner W R, Beaufort M F, Rousselet S and Horstmann J T 2005 Appl. Phys. Lett. 86252109

[7] Hutchinson J W, Thouless M D and Liniger E G 1992 Acta Metall. Mater. 40 295-308

[8] Jagla E A 2007 Phys. Rev. B 75085405

[9] Colin J, Coupeau C, Durinck J and Grilhe J 2009 Epl 8654002

[10] Audoly B 1999 Phys. Rev. Lett. 83 4124-7

[11] Peyla P 2000 Phys. Rev. E 62 R1501-R4

[12] Goudeau P, Villain P, Tamura N and Padmore H A 2003 Appl. Phys. Lett. 83 51-3

[13] Okba F, Cherkashin N, Di Z, Nastasi M, Rossi F, Merabet A and Claverie A 2010 Appl. Phys. Lett. 97031917

[14] Shao L, Lin Y, Lee J K, Jia Q X, Wang Y, Nastasi M, Thompson P E, Theodore N D, Chu P K, Alford T L, Mayer J W, Chen P and Lau S S 2005 Appl. Phys. Lett. 87091902

[15] Hochbauer T, Misra A, Hattar K and Hoagland R G 2005 J. Appl. Phys. 98123516

[16] Nan L, Martin M S, Anderoglu O, Misra A, Shao L, Wang H and Zhang X 2009 J. Appl. Phys. 105 123522

[17] Demkowicz M J, Bhattacharyya D, Usov I, Wang Y Q, Nastasi M and Misra A 2010 Appl. Phys. Lett. 97161903

[18] Pelizzo M G, Corso A J, Zuppella P, Windt D L, Mattei G and Nicolosi P 2011 Opt. Express 19 14838-44

[19] Nedelcu I, van de Kruijs R W E, Yakshin A E, Tichelaar F, Zoethout E, Louis E, Enkisch H, Muellender S and Bijkerk F 2006 Thin Solid Films 515 434-8

[20] de Rooij-Lohmann V I T A, Kozhevnikov I V, Peverini L, Ziegler E, Cuerno R, Bijkerk F and Yakshin A E 2010 Appl. Surf. Sci. 256 5011-4

[21] Nedelcu I, van de Kruijs R W E, Yakshin A E and Bijkerk F 2008 J. Appl. Phys. 103083549

[22] Zoethout E, Sipos G, van de Kruijs R, Yakshin A, Louis E, Muellender S and Bijkerk F 2003 Proc. SPIE 5037872 